Document downloaded from:

http://hdl.handle.net/10251/37713

This paper must be cited as:

Barrachina, J.; Garrido, P.; Fogue, M.; Martínez, FJ.; Cano Escribá, JC.; Tavares De Araujo Cesariny Calafate, CM.; Manzoni, P. (2012). VEACON: A Vehicular Accident Ontology designed to improve safety on the roads. Journal of Network and Computer Applications. 35(6):1891-1900. doi:10.1016/j.jnca.2012.07.013.

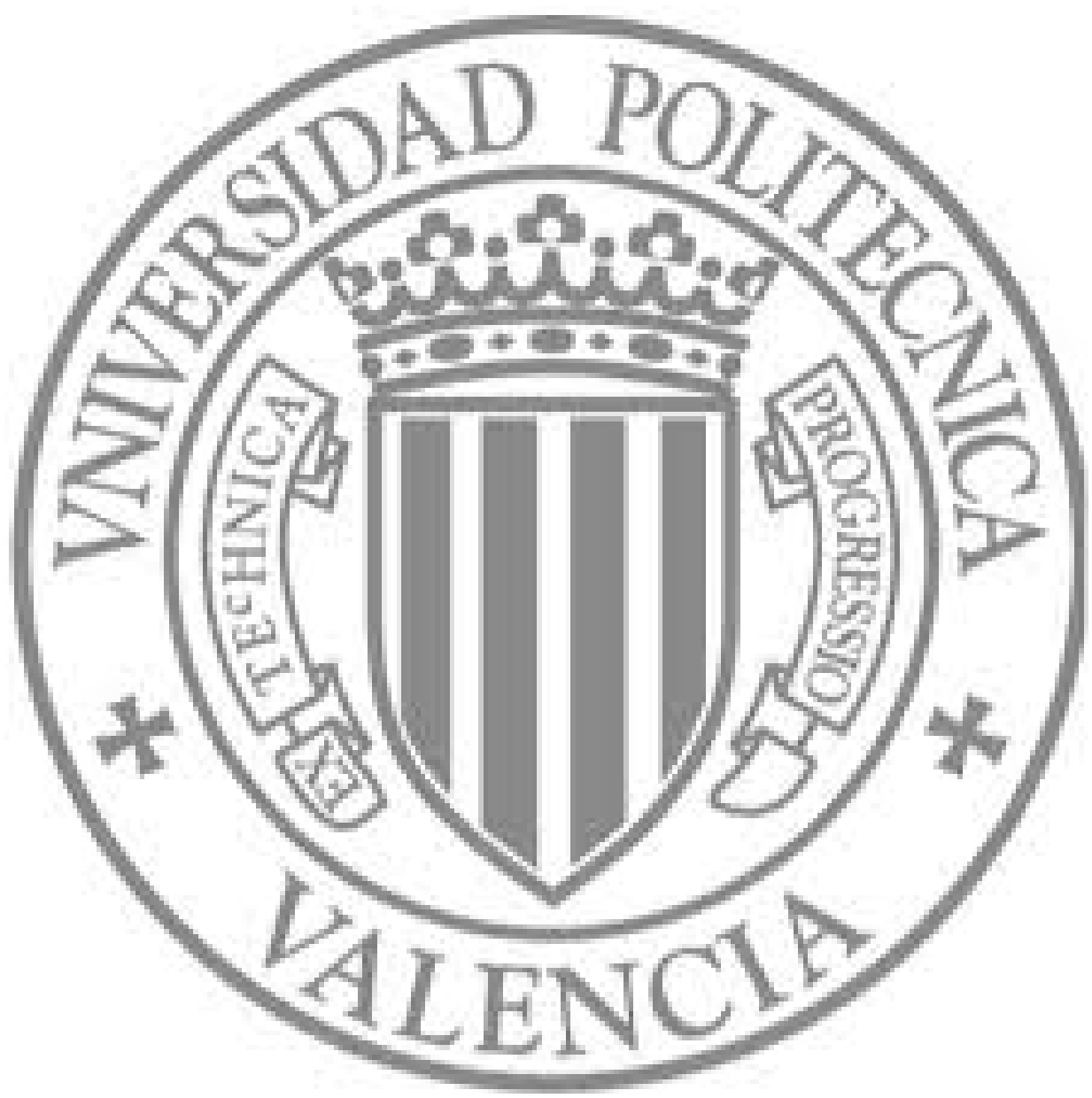

The final publication is available at

hppt://dx.doi.org/10.1016/j.jnca.2012.07.013

Copyright Elsevier 


\title{
VEACON: a VEhicular ACcident ONtology Designed to Improve Safety on the Roads
}

\author{
Javier Barrachina, Piedad Garrido, Manuel Fogue, Francisco J. Martinez \\ University of Zaragoza, Spain \\ Email: \{barrachina, piedad, m.fogue,f.martinez\}@unizar.es \\ Juan-Carlos Cano, Carlos T. Calafate, Pietro Manzoni \\ Universitat Politècnica de València, Spain \\ Email: \{jucano, calafate, pmanzoni\}@disca.upv.es
}

\begin{abstract}
Vehicles are nowadays provided with a variety of new sensors capable of gathering information about themselves and from their surroundings. In a near future, these vehicles will also be capable of sharing all the harvested information, with the surrounding environment and among nearby vehicles over smart wireless links. They will also be able to connect with emergency services in case of accidents. Hence, distributed applications based on Vehicular Networks (VNs) will need to agree on a 'common understanding' of context for interoperability, and, therefore, it is necessary to create a standard structure which enables data interoperability among all the different entities involved in transportation systems. In this paper, we focus on traffic safety applications; specifically, we present the VEhicular ACcident ONtology (VEACON) designed to improve traffic safety. The instances of our ontology are composed by the information collected when an accident occurs, and the data available in the General Estimates System (GES) accidents database. We assess the reliability of our proposal using both realistic crash tests, and vehicular network simulations, based on the ns- 2 simulation tool. Experimental results highlight that both nearby vehicles and infrastructure elements (RSUs) are notified about and accident in just a few seconds, increasing the emergency services notification effectiveness.
\end{abstract}

Keywords: Vehicular Networks, Ontologies, Intelligent Transportation Systems (ITS), Vehicular Accidents, VANETs 


\section{Introduction}

Currently, one of the most important factors of globalization is transportation. Although the purpose of transport has not changed with globalization, the factors triggering the emergence of a global transportation system (e.g. volume, capacity, speed, and efficiency) have evolved. Moving goods and people as quickly as possible all around the world requires advanced integrated transportation systems (Di Lecce and Amato, 2009). Information Technology (IT) and transport infrastructure help to manage transportation systems in an accurate and effective manner. Intelligent Transportation Systems (ITS) will play a leading role in our society, especially in scenarios such as warning drivers about vehicle accidents in real time, efficiently managing vehicle information required by governments and authorities, or even being able to offer drivers a variety of added services.

The specific characteristics of Vehicular Networks favor the development of attractive and challenging services and applications. However, distributed applications based on Vehicular Networks need to agree on a 'common understanding' of context for interoperability on a contextual level. We consider that some semantic web ideas can be applied to modern transportation systems to build up such context. The Semantic Web (Berners-Lee et al., 2001) is an extension of the traditional web which allows machines to interpret the meaning of data thanks to the use of ontologies. An ontology is a description of a small part of the real world, including the types of items that appear in this world, the relations among them, the leading elements, and their restrictions. Typically, an ontology is defined as a formal specification of conceptualization (Gruber, 1995).

In this paper we focus on safety applications. Specifically, our aim is to improve traffic safety by using an ontology-based approach in Vehicular Networks. To that end, we propose the VEhicular ACcident ONtology (VEACON), a novel lightweight ontology proposed with the aim of successfully sharing and reusing knowledge about traffic accidents. VEACON allows to efficiently structure and encode the information collected by sensors in the vehicle, enabling the interoperatibility among all the agents involved in modern ITS (i.e., vehicles, RSUs, emergency services, and authorities).

Nowadays, vehicular networking technologies allow a vehicle to alert emergency services in case of an accident. Although there are many solutions that 
relay on Vehicular Networks for that purpose, there are fewer solutions based on semantics to send accident information to the emergency services. In this paper, we explore the use of a formal ontology framework for sending critical information captured by vehicles involved in road accidents. This information will not only be sent to the emergency services, but also it will be shared among the nearby vehicles. Hence, this warning information will be used for different proposes such as: (a) preventing new accidents (avoiding that other vehicles collide with the vehicles already involved in the accident), (b) helping to allocate resources for a rescue, and (c) maintaining statistics on road accidents, which allows fast database searches and the creation of prediction models to estimate the severity of future accidents. This estimation could be done with data mining classification models by combining the proposed ontology with existing databases (Chong et al., 2005).

This paper is organized as follows: Section 2 reviews the related work regarding the use of ontologies applied on ITS. Section 3 presents VEACON, our proposed ontology. In Section 4, we assess the feasibility our proposal by doing some real experiments as well as carrying out some simulation tests. Finally, Section 5 concludes this paper.

\section{Related Work}

For the proper operation of traffic safety systems, we must consider two different factors: (i) vehicles must be able to communicate among them in order to share information, and (ii) the shared information should be understood by all the entities involved in transportation systems. The first factor has been widely studied by the wireless networking research community (Martinez et al., 2010b; Bakhouya et al., 2011; Antolino Rivas et al., 2011; Daeinabi et al., 2011). However, the second factor has not been studied to the same extent.

Regarding the use of semantics in vehicular environments, some authors have worked on the integration of transportation systems information and semantics. Zhai et al. (2008b) presented an ontology for structuring data traffic. Zhai et al. (2008a) introduced a knowledge navigation system with urban traffic information based on the XML Topic Maps technology, enabling intelligent information retrieval through association between topics. These different works highlight the importance of using ontologies in ITS, however they do not provide any ontology specially designed for ITS safety. 
Regarding ITS safety, Eigner and Lutz (2008) showed the need for ontological context models for VNs safety environments, and how all the components of the system would be able to understand one another through these models. They considered that vehicles should incorporate a variety of sensors to get data from the vehicles themselves, as well as from their surroundings. In addition, information obtained by these sensors could be shared with other vehicles using VNs. The authors showed that vehicular applications can benefit from the inherent characteristics of ontological models such as distributed composition, partial validation, richness and quality of information, as well as a certain level of formality. Additionally, authors proved that calculations on the model are still fast enough to fulfill real-time requirements imposed by the active safety systems of vehicles. However, they did not build a specific ontology. More recently, Kannan et al. (2010) proposed an ontology modelling approach for assisting vehicle drivers through warning messages during time critical situation. Authors focused on generating the alert messages based on the context aware parameters such as driving situations, vehicle dynamics, driver activity, and the environment.

Although all the above presented works proposed ontological models for warning messages using Vehicular Networks, none of them enriched their proposal with historical information to estimate the severity of accidents.

\section{VEACON: Our Ontology for Vehicular Networks}

From the point of view of Communications and Information Technologies for Vehicular Networks, ITS applications will rely on efficient vehicular communications and smart exchange of information among all the entities involved, i.e., vehicles, RSUs, emergency services, management authorities and police. When a traffic accident occurs, a crucial issue that should be addressed in transportation systems is to collect as much information as possible, since vehicles should rapidly warn nearby vehicles and the emergency services to obtain a quick and efficient response from them. However, the information usually collected in accidents is neither structured nor does it present relationships between their basic elements. We propose to organize this information by using an approach based on the Semantic Web, where the information can be obtained through various techniques such as ontologies, classifications, taxonomies, thesauri, or Topic Maps (Garshol, 2004). We consider that the use of ontologies is the more common and versatile technique to organize such kind of contents. 


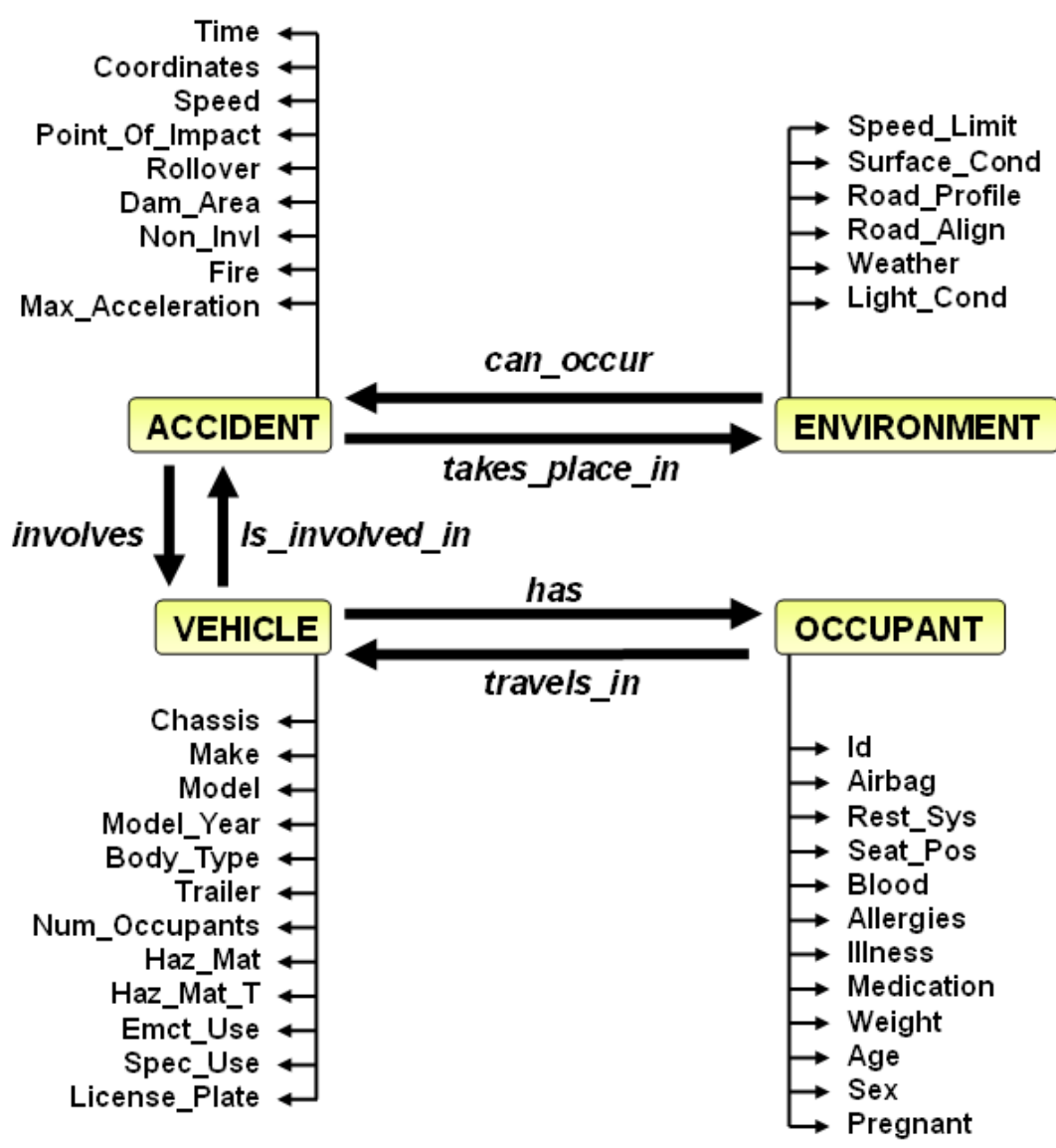

Figure 1: VEACON ontology components.

Our system gets the information from warning messages exchanged among vehicles and emergency services. This information should be, on the one hand, concise enough to avoid irrelevant information, but, on the other hand, it should not ignore any information that might be useful for the emergency services to determine the most suitable set of resources. Thus, the delivered information should include: data about the conditions under which the accident occurred, data about the occupants of the vehicle, as well as a description of the security systems included within the vehicle. These data will be sent to the emergency services to provide a more detailed view of the conditions of the accident before their arrival.

In this work, we use an ontology based technique to group all these information sources, while allowing to make inferences over the collected data. 
An ontology formally represents knowledge, and it can be used to reason about the entities within that domain, and may be used to describe the domain, so we can elaborate estimations about different factors of the accident (impact severity, passenger injuries, and so on). Basically, an ontology consists of three parts: classes and instances of real-world items, relations among these items, and rules for modeling knowledge and complex behaviors (creation, restraint and response). Specifically, we propose the Vehicle Accident Ontology (VEACON), a novel lightweight ontology proposed with the aim of sharing and reusing knowledge about the vehicles involved in road accidents. VEACON meets our requirements since it: (i) promotes interoperability between different knowledge bases, (ii) provides an infrastructure or cooperative system, (iii) facilitates the information sharing, and (iv) enables domain knowledge reuse. VEACON consists of a set of classes representing the categories of the entities of interest in the ITS domain, the attributes which define properties of those classes, and the relationships between those entities.

Figure 1 shows the basic VEACON lightweight ontology structure, which groups the available information into four different areas: Vehicle, Accident, Occupant and Environment. As for the languages, we decided to use the Ontology Web Language (OWL) ${ }^{1}$ to create XML-based messages, since it is a flexible and expressive language which provides a basic syntax to describe the relationships between entities. Listing 1 shows an example of a VEACONcompliant warning message.

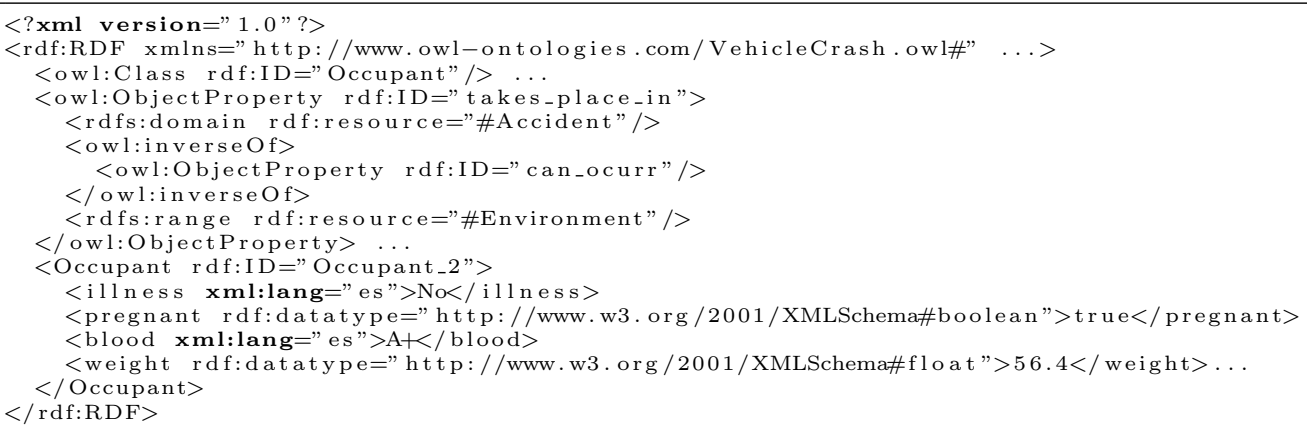

Listing 1: Example of a VEACON OWL-based warning message.

\footnotetext{
${ }^{1}$ http://www.w3.org/TR/owl-ref
} 
Due to privacy requirements of the collected data, especially the medical information of the passengers, all the messages generated with the VEACON ontology are encrypted using the Advanced Encryption Standard (AES) (Daemen and Rijmen, 2002) before being sent by the vehicles.

\subsection{Getting information into our Ontology}

Nowadays, vehicles incorporate a series of sensors to obtain information about different areas. Examples of them are crush zone crash sensors (Breed, 1991), occupant position sensors (Breed et al., 1998), rain sensors (Petzold, 1999), or seat belt tension sensors (Husby and Simpson, 2000). Therefore, it is possible to get key information from these sensors when an accident occurs.

Furthermore, we consider that future vehicles will get additional information from the environment and its occupants, since vehicles will be provided with sensors capable of knowing if there are pedestrians or cyclists involved in an accident, and also information regarding the health of the occupants such as blood group or heart problems. Currently, there are some approaches addressing these issues; for example, the Ford Motor Company (2011) is designing seats that can monitor the driver's heartbeat in real time. For personal information and health data of the occupants, we consider that each occupant could have this information on his cell phone, and the vehicle would collect this data when boarding.

To allow estimating the severity of the accidents, our proposal also uses the General Estimates System (GES), an historical database maintained by the (National Highway Traffic Safety Administration (NHTSA), 2011), which contains information related to previous traffic accidents, obtained from a sample of Police Accident Reports (PARs) collected all over the USA roads. To protect individual privacy, no personal information such as names, addresses or specific crash location is coded.

\subsection{VEACON Fields}

For our proposed ontology, we selected a number of existing fields in the GES database, and we have also added others that we felt necessary. The selection was specifically made considering the data that can be significant when an accident occurs.

We have grouped the information into four areas: (i) Vehicle, which contains the characteristics of the vehicle and data for identification; (ii) Accident, which collects the location and time of the crash, the characteristics 
Table 1: Vehicle dataset.

\begin{tabular}{|l|l|}
\hline \hline Field & Description \\
\hline Chassis & Vehicle chassis number \\
\hline Make & Manufacturer of the vehicle \\
\hline Model & Vehicle model \\
\hline Model_Year & Vehicle model year \\
\hline Body_Type & Vehicle body type \\
\hline Trailer & If vehicle is towing trailing units \\
\hline Num_occupants & Number of vehicle occupants \\
\hline Haz_Mat & If vehicle is carrying hazardous materials \\
\hline Haz_Mat_T & Hazardous materials type \\
\hline Emcy_use & If vehicle is on an emergency run \\
\hline Spec_use & Vehicle special use category applied \\
\hline License_Plate & Vehicle plate number \\
\hline \hline
\end{tabular}

of the collision, and the caused damage; (iii) Occupant, which collects occupants' personal and medical information, their location within the vehicle, and the safety systems deployed; and (iv) Environment, which contains information about road, weather and lighting conditions.

In the set of data related to Vehicle, the fields used are those indicated in Table 1. For this dataset, we used available fields from the GES database, and added two new fields: License_Plate and Chassis. We believe that they are necessary since they provide a unique identifier (Chassis), and allow the emergency services to quickly recognize vehicles at the scene of accident (License_Plate).

In the set of data related to Accident, the fields used are those indicated in Table 2. For this dataset, we used fields from the Accident and Vehicle dataset from the GES database, and added two new fields: Coordinates and Max_Acceleration. We consider that they are useful to locate the crash site (Coordinates), and to obtain a measure of the impact severity (Max_Acceleration). Note that, if using the appropriate technology, the system can also determine the value of the Non_Invl field, which indicates whether people outside the vehicle (pedestrian or cyclist) were involved in the crash. This information could be very useful for emergency services to decide the rescue resources required. We did not include a field indicating 
Table 2: Accident dataset.

\begin{tabular}{|l|l|}
\hline \hline Field & Description \\
\hline Time & Time when crash occurred \\
\hline Coordinates & Crash point coordinates \\
\hline Speed & Vehicle speed at the crash moment \\
\hline Point_Of_Impact & Point of impact for the crashed vehicle \\
\hline Rollover & If vehicle has overturned \\
\hline Dam_Area & Dam area for the crashed vehicle \\
\hline Non_Invl & Number of non-motorists involved in the crash \\
\hline Fire & If vehicle is in fire \\
\hline Max_Acceleration & Vehicle maximum deceleration during the crash \\
\hline \hline
\end{tabular}

the number of vehicles involved in the crash because all the collided vehicles will send their own messages.

In the set of data related to Occupants, the fields used are those indicated in Table 3. For this dataset, we used basic fields from the GES database, and also added nine new fields: Blood, Allergies, Illness, Medication, Pregnant, Weight, Age, Sex and Id. The first eight fields could be previously stored on the mobile phone of each passenger. Then, in case of an accident, emergency services will receive all this individualized medical information, thereby allowing emergency services to identify each person.

Finally, Table 4 shows the different fields related to Environment. For this dataset, we used fields from the Accident dataset available in the GES database.

\subsection{Qualitative Comparison of Similar Existing Ontologies}

Table 5 presents a summary of the VEACON comparison we made with respect to other existing ITS ontologies. We have structured the comparison in eight different categories: (a) the source of their attributes, (b) if they used any historical database, (c) if they support accident severity prediction, (d) the tagging language used, (e) the software frameworks used, (f) if data is grouped into classes, $(\mathrm{g})$ if they present the relationships, and (h) the method selected for the evaluation.

As shown, VEACON is the only ontology that uses historical data for its design, enabling the prediction of accidents severity, which in our opinion makes the difference, since nowadays traffic accidents cause millions of 
Table 3: Occupant dataset.

\begin{tabular}{|l|l|}
\hline \hline Field & Description \\
\hline Id & Occupant identifier \\
\hline Airbag & Airbag availability/function in the seat position of the occupant \\
\hline Rest_Sys & Restraints that are being used by the occupant immediately prior to the crash \\
\hline Seat_Pos & Occupant seating position \\
\hline Blood & Occupant blood type \\
\hline Allergies & Occupant allergies \\
\hline Illness & Occupant illness \\
\hline Medication & If occupant needs specific medication or treatment \\
\hline Weight & Occupant weight \\
\hline Age & Occupant age \\
\hline Sex & Occupant gender \\
\hline Pregnant & If occupant is pregnant \\
\hline \hline
\end{tabular}

people killed or severely injured. Moreover, in contrast to VEACON, the rest of studied ontologies have not been evaluated under real testbed crash environments, and using vehicular simulations.

\section{Validation of Our VEACON Proposal}

In vehicular environments, wireless technologies enable peer-to-peer mobile communication among vehicles $(\mathrm{V} 2 \mathrm{~V})$ and communication between vehicles and the infrastructure (V2I). We think that the combination of V2V

Table 4: Environment dataset.

\begin{tabular}{|l|l|}
\hline \hline Field & Description \\
\hline Speed_Limit & Roadway legal speed limit \\
\hline Surface_Cond & Roadway surface condition \\
\hline Road_Profile & Roadway profile \\
\hline Road_Align & Roadway alignment \\
\hline Weather & Atmospheric conditions at the time of the accident \\
\hline Light_Cond & Light conditions at the time of the accident \\
\hline \hline
\end{tabular}


Table 5: ITS Ontologies Comparison.

\begin{tabular}{|c|c|c|c|}
\hline Description & VEACON & $\begin{array}{l}\text { Eigner and Lutz } \\
(2008)\end{array}$ & $\begin{array}{l}\text { Kannan et al. } \\
(2010)\end{array}$ \\
\hline $\begin{array}{l}\text { Where does it select } \\
\text { attributes? }\end{array}$ & GES database enriched & At their own discretion & At their own discretion \\
\hline $\begin{array}{l}\text { There is historical } \\
\text { data to compare ac- } \\
\text { cidents? }\end{array}$ & Yes, the GES database & No & $\begin{array}{l}\text { No, it is designed to } \\
\text { support a Driver Assis- } \\
\text { tance System }\end{array}$ \\
\hline $\begin{array}{l}\text { Does predict it the } \\
\text { damage from acci- } \\
\text { dent? }\end{array}$ & $\begin{array}{l}\text { Yes, using historical } \\
\text { data }\end{array}$ & $\begin{array}{l}\text { No, it is only designed } \\
\text { to prevent accidents }\end{array}$ & $\begin{array}{l}\text { This ontology is not } \\
\text { specific for traffic acci- } \\
\text { dents }\end{array}$ \\
\hline Tagging language & OWL & OWL & OWL \\
\hline Software used & Protégé & Not specified & Protégé \\
\hline $\begin{array}{l}\text { Does it present } \\
\text { the ontology } \\
\text { relationships? }\end{array}$ & Yes & No & Only partially \\
\hline System Evaluation & $\begin{array}{l}\text { Crash tests and net- } \\
\text { work simulations }\end{array}$ & $\begin{array}{l}\text { Simulations using the } \\
\text { Virtual Traffic Simula- } \\
\text { tor (VISSIM) }\end{array}$ & Ad-hoc simulator \\
\hline $\begin{array}{l}\text { Map Topology for } \\
\text { Validation }\end{array}$ & Real roadmaps & Synthetic roadmaps & $\begin{array}{l}\text { Synthetic single, 2-way, } \\
\text { and } 4 \text {-way roads }\end{array}$ \\
\hline
\end{tabular}

and V2I communications can propel our communication capabilities even further, improving the traffic safety under Intelligent Transportation Systems (ITS). To verify that our ontology works correctly in Vehicular Networks, we performed two different kinds of experiments: (i) real crash tests involving Vehicle-to-Infrastructure (V2I) communications, to verify that the message using our ontology proposal is correctly sent to the emergency services in case of an accident, and (ii) vehicular network simulations, to study how VEACON messages would be propagated to the rest of vehicles in terms of V2I and V2V communications, in a realistic urban environment.

\subsection{Real Crash Tests}

To prove the feasibility of our ontology, we performed several crash experiments in the facilities of Applus+ IDIADA Passive Security Department 
sited in Santa Oliva (Tarragona, Spain) ${ }^{2}$. This laboratory is one of the most sophisticated crash test laboratories in the world, and is an official center for approval under the EuroNCAP: European New Car Assessment Programme (2011). Due to the cost of using real vehicles in the collision experiments, tests were performed using a platform (known as "sled") which is able to simulate different kind of vehicles and impact severities in traffic accidents.

Figure 2 shows the sled used in our tests. As shown, a series of weights were added to accurately simulate the behavior of a conventional vehicle. Figure 3 details the electronic components used to implement the OBU prototype on the platform. Validation experiments consisted in front, side and rear-end collision tests with different severities. The classification of the severity of the collision is dictated by the EuroNCAP and RCAR tests (RCAR, the Research Council for Automobile Repairs, 2011). In our experiments, the On Board Unit (OBU) installed in the sled collected all the information provided by the sensors, built the warning message according to our VEACON ontology, and sent this alert information at the collision time by using wireless communications. An external computer acted as a Road Side Unit (RSU), in charge of receiving the warning messages broadcasted by vehicles, and forwarding them to a suitable Public Safety Answering Point (PSAP) or 112 Service Center.

The results obtained in the real crash tests were very promising. Figure 4 shows some of the acceleration pulses recorded by the OBU and sent to the RSU for three different front crash tests. Although different types of vehicles were tested, the figure only includes those corresponding to the large family car segment. The OBU is in charge of determining the severity of the direct impact, but interpreting acceleration values is not trivial since the received pulses have a very limited duration, and also because both their amplitude and duration should be considered in the classification. As shown in Figure 4, using simple acceleration thresholds to distinguish acceleration pulses is not enough (e.g. the minor accident has a peak deceleration that is greater than for the severe accident). However, we discovered that the value of the integral function defined as the variation of acceleration over time allows simple and accurate pulse classification since it accounts for both amplitude and duration of the pulse.

The experiments performed in real crash tests proved that our system was

\footnotetext{
${ }^{2}$ http://www.idiada.es
} 


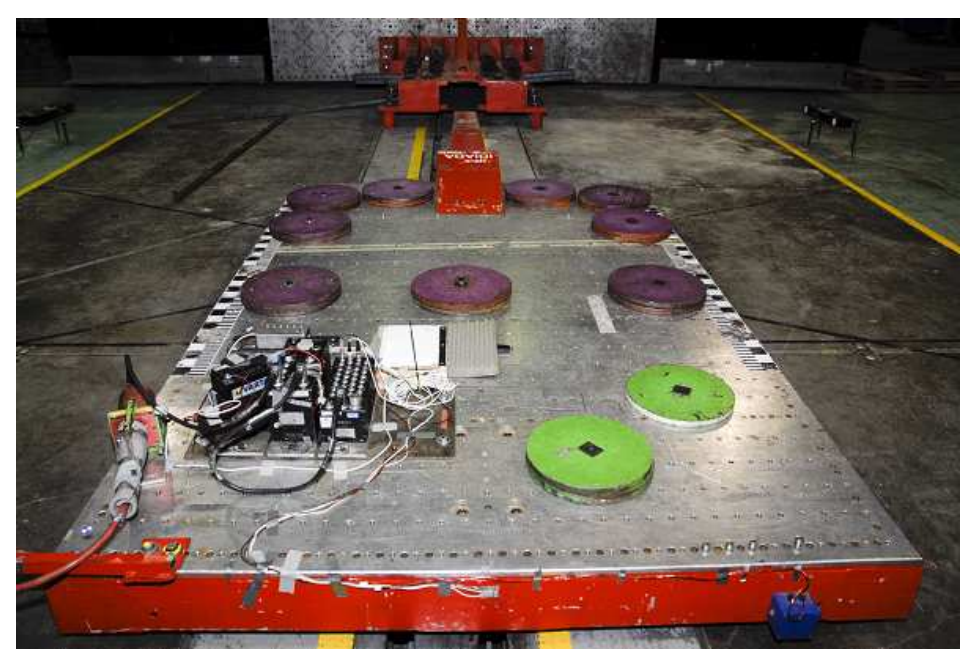

Figure 2: Sled used in our crash tests.

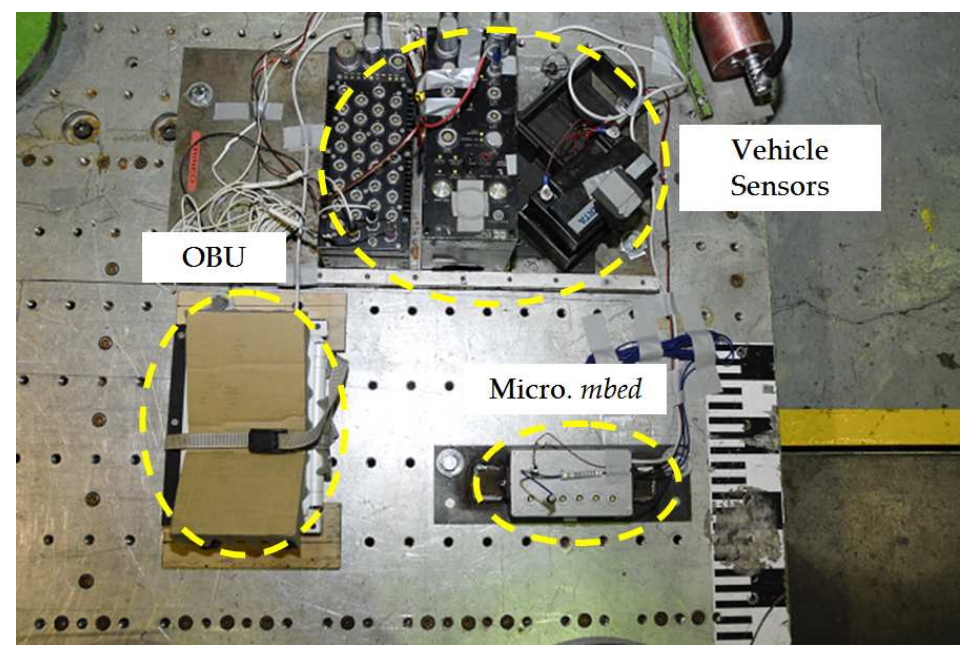

Figure 3: Close-up of the electronic components installed on the sled. 


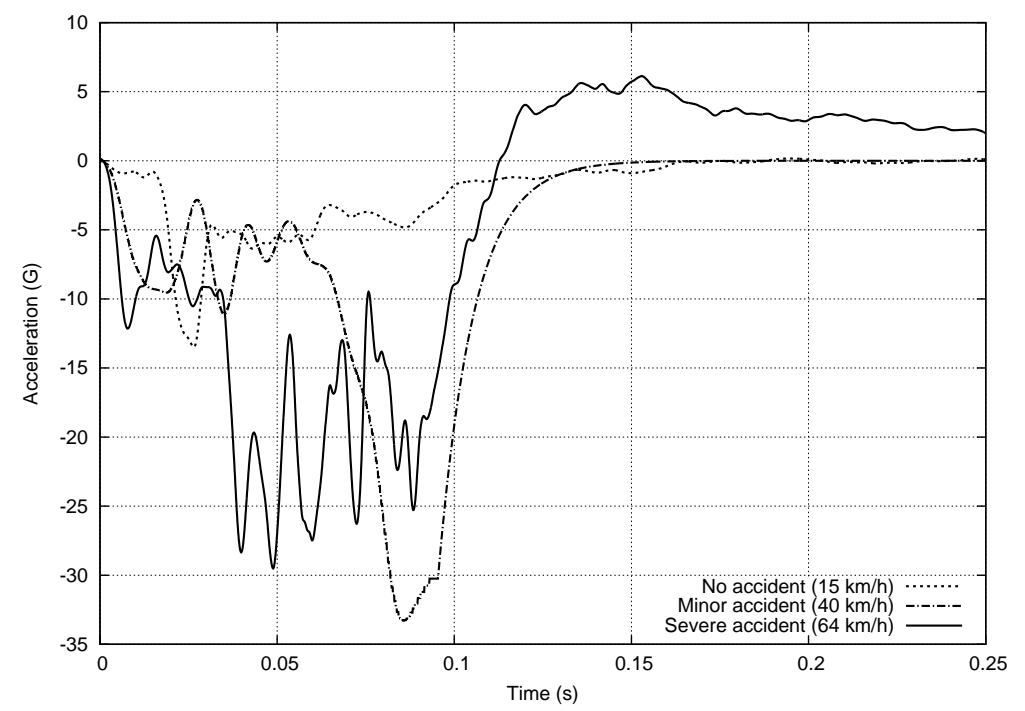

Figure 4: Vehicle acceleration pulses during front crashes in the large family car segment.

able to collect all the information provided by in-vehicle sensors, to build the VEACON-compliant message, and to communicate with the RSU in every tested situation without message loss. Moreover, the OBU was able to accurately determine the impact severity by using the integral approach in all cases, generating an adequate warning message, and sending it to the nearest RSU. Warning messages were broadcasted and successfully received by the RSU, and the contained information was correctly extracted and interpreted (see Figure 5). Consequently, we conclude that the VEACON ontology can be successfully used to notify accident situations in real environments.

\subsection{Network Simulation Tests}

To study how messages built using VEACON propagate in a vehicular network scenario, simulations were done using the ns-2 simulator. We improved the simulator by including the IEEE 802.11p standard closely, which defines enhancements to 802.11 required to support ITS applications (IEEE 802.11 Working Group, 2010). In terms of the physical layer, the data rate used for message broadcasting is $6 \mathrm{Mbit} / \mathrm{s}$, as this is the maximum rate for broadcasting in $802.11 \mathrm{p}$. The MAC layer was also extended to include four different priorities for channel access. Therefore, application messages are categorized into four different Access Categories (ACs), where AC0 has the lowest and AC3 the highest priority. 


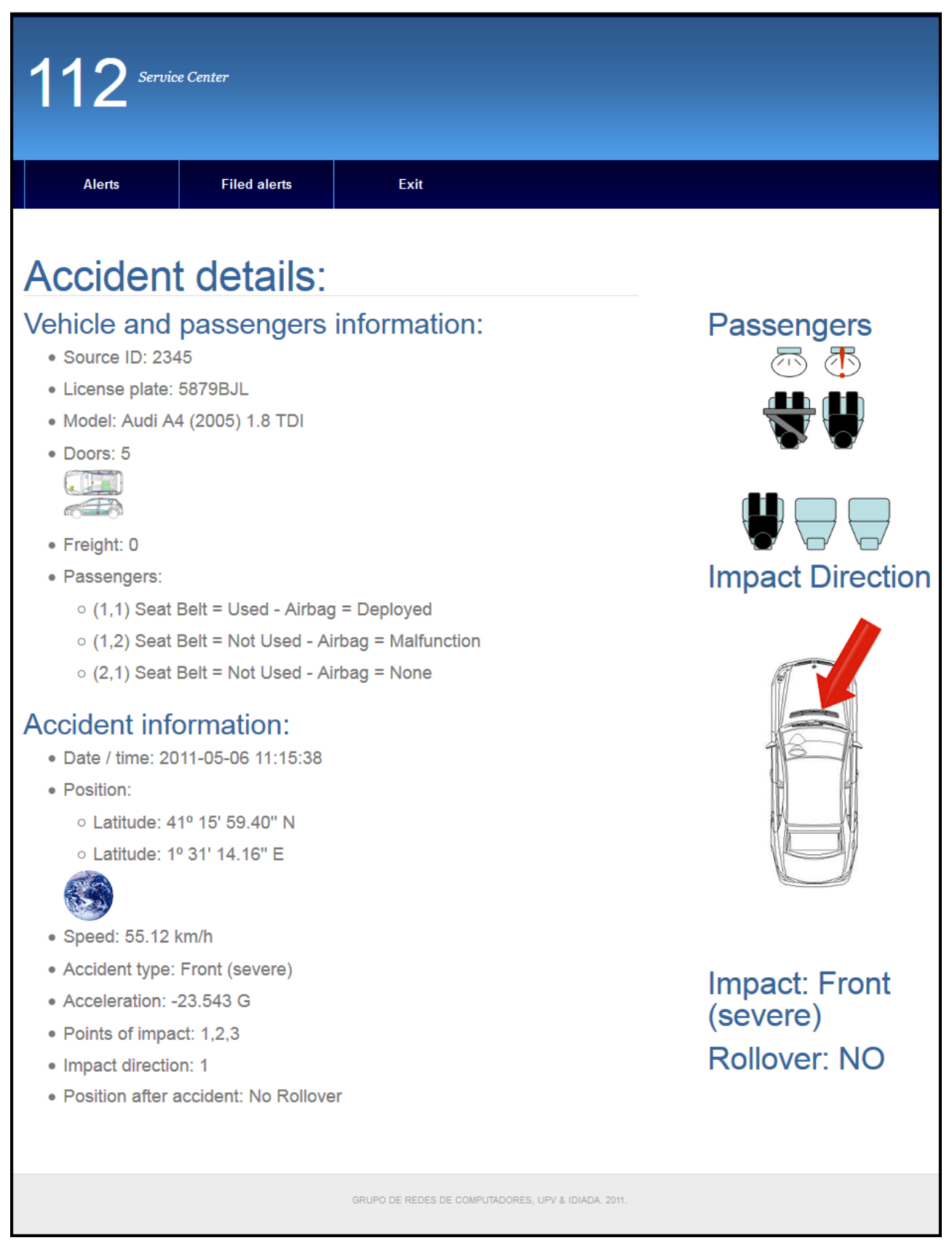

Figure 5: VEACON-compliant information received by the 112 Service Center and presented in a web interface. 
The purpose of the 802.11 p standard is to provide the minimum set of specifications required to ensure interoperability between wireless devices attempting to communicate in potentially rapid changing communication environments. For our simulations, we chose the IEEE $802.11 \mathrm{p}$ because it is expected to be widely adopted by the industry.

We want to evaluate whether or not our proposal ontology could affect to the dissemination of warning messages in Vehicular Networks.

We tested our proposed ontology by evaluating the performance of a Warning Message Dissemination mechanism where each vehicle periodically broadcasts information about itself, or about an accident. These messages are built according to our VEACON ontology.

Our simulations have been carried out in two different scenarios of $4 \mathrm{~km}^{2}$, obtained from real maps from New York (USA) and Rome (Italy). As shown in Figure 6, the New York map presents the longest streets, mostly arranged in a Manhattan-grid style, while the city of Rome represents the opposite situation, with short streets in a highly irregular layout.

To increase the realism of our simulations, we used Citymob for Roadmaps $(\mathrm{C} 4 \mathrm{R})^{3}$, a mobility generator based on SUMO (Krajzewicz and Rossel, 2007). $\mathrm{C} 4 \mathrm{R}$ includes all the original characteristics from SUMO (collision-free vehicle movement, multi-lane streets, etc.). In addition, it is able to define attraction and repulsion points which simulate areas with different vehicle densities, something very common in real cities. Regarding the radio propagation model, the network simulator was also modified to make use of our Real Attenuation and Visibility (RAV) scheme (Martinez et al., 2010a), which proved to increase the level of realism in VANET simulations since it accounts for the effect of obstacles (e.g., buildings) in radio signal propagation when simulating urban scenarios.

We simulated a frontal impact scenario where two vehicles are involved. The first vehicle is a family car with two occupants, and expressing all the information required, according to VEACON, a message of 13 KBytes. The second vehicle is a minivan with eight occupants, which required up to 18 KBytes to code the data for all passengers. Each simulation run lasted for 450 seconds. In order to achieve a stable state, we collect data only after the first 60 seconds. All results represent an average of over 30 executions with different scenarios (maximum error of $10 \%$ with a degree of confidence

\footnotetext{
${ }^{3} \mathrm{C} 4 \mathrm{R}$ is available at http://www.grc.upv.es/software/
} 


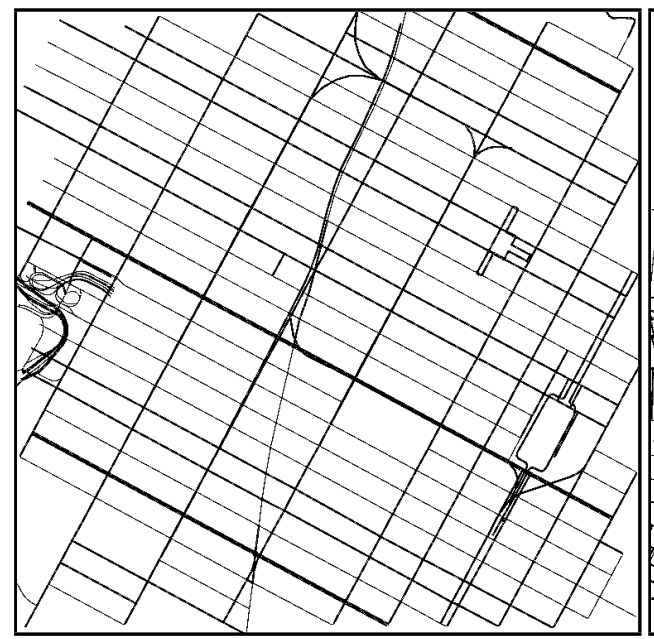

(a)

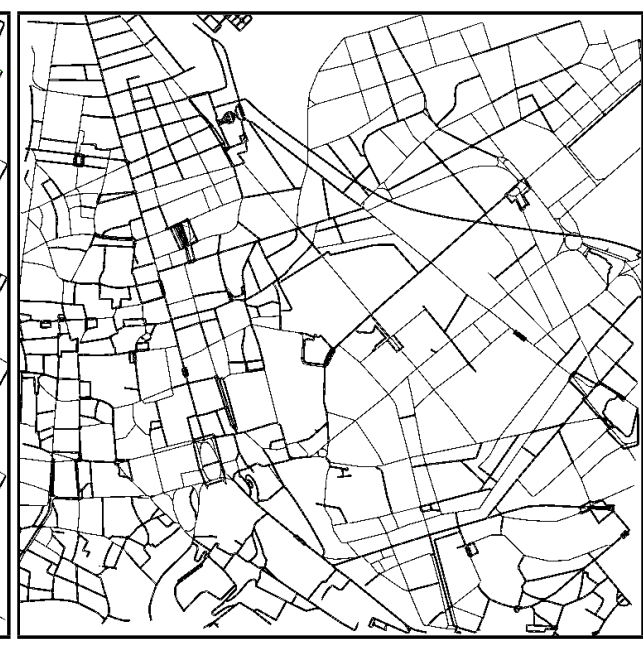

(b)

Figure 6: Scenarios used in our simulations: (a) fragment of the city of New York (USA), and (b) fragment of the city of Rome (Italy).

of $90 \%$ ). Table 6 shows the parameters used in the simulations.

In order to determine the feasibility of VEACON in different situations, we present the results obtained when considering both V2I and V2V communications. We consider that some factors, such as the density of vehicles, the density of RSUs, or the map topology, should have a significant impact on the performance of our ontology-based warning message dissemination scheme. Therefore, we performed different experiments by varying these factors, and studied their effect on the following metrics: (i) the notification time (i.e., the period elapsed between the time when a warning-mode vehicle requests for help, and the time when any RSU receives the warning message, delivering it to the next Public Safety Answering Point (PSAP) or 112 Service Center), (ii) the percentage of RSUs receiving the warning messages, (iii) the warning notification time (i.e., the time required by nearby vehicles to receive a warning message sent by a collided vehicle), and (iv) the percentage of vehicles receiving the warning messages. These metrics are crucial when assessing with the usefulness of our studied system, since a warning message delivered too late is useless when facing dangerous situations, and nearby vehicles must be informed about these situations. 
Table 6: Parameter Values for the Simulations.

\begin{tabular}{|l|c|}
\hline \hline Parameter & Value \\
\hline number of vehicles & $50,100,200$, and 400 \\
simulated cities & New York and Rome \\
simulated area & $2000 \mathrm{~m} \times 2000 \mathrm{~m}$ \\
number of collided vehicles & 2 \\
warning packet size & 13 and $18 \mathrm{~K} B$ \\
packets sent by vehicles & 1 per second \\
warning message priority & $A C 3$ \\
normal message priority & $A C 1$ \\
mobility generator & $C 4 R$ \\
mobility models & Krauss and Downtown \\
MAC/PHY & $802.11 \mathrm{p}$ \\
radio propagation model & RAV \\
maximum transmission range & $400 \mathrm{~m}$ \\
\hline \hline
\end{tabular}

\subsubsection{V2I Communications Results}

Regarding V2I communications, Table 7 shows the minimum notification time and the reachability (i.e., the percentage of times that warning messages reach any RSU), when varying the number of vehicles, the number of RSUs, and the simulated roadmap. As shown, the simulated roadmap affects both the warning notification time and the percentage of RSUs receiving the warning messages, especially when the vehicle density is very low (Rome shows higher notification times, but, in contrast, it shows a higher percentage of successful RSU notifications). When 400 vehicles are simulated, notification time is slightly higher in Rome, since the topology is more complex than New York. However, results show that in complex roadmaps like Rome, the percentage of receiving RSUs is higher compared to New York. We think that this demonstrates that V2I communications can play an important role in such complex scenarios. Moreover, as expected, results reflect that increasing the density of vehicles highly increases the chances for warning messages to reach any RSU, i.e., the emergency services notification effectiveness.

\subsubsection{V2V Communications Results}

Regarding V2V communications, Figure 7 shows the obtained results when varying the scenario topology and the vehicle density. 
Table 7: V2I Simulation Results.

\begin{tabular}{|c|c|c|c|c|c|}
\hline \multirow[b]{2}{*}{ Vehicles } & \multirow[b]{2}{*}{ RSUs } & \multicolumn{2}{|c|}{ New York } & \multicolumn{2}{|c|}{ Rome } \\
\hline & & Notif. time (s) & Reach. (\%) & Notif. time (s) & Reach. (\%) \\
\hline \multirow{4}{*}{50} & 1 & 0.383 & 5 & 12.919 & 25 \\
\hline & 2 & 0.674 & 5 & 6.669 & 55 \\
\hline & 4 & 0.790 & 30 & 6.188 & 65 \\
\hline & 8 & 0.790 & 30 & 4.841 & 75 \\
\hline \multirow{4}{*}{100} & 1 & 1.383 & 50 & 1.724 & 95 \\
\hline & 2 & 1.146 & 65 & 1.427 & 95 \\
\hline & 4 & 1.107 & 70 & 1.456 & 95 \\
\hline & 8 & 1.247 & 70 & 1.283 & 95 \\
\hline \multirow{4}{*}{200} & 1 & 1.147 & 80 & 1.643 & 100 \\
\hline & 2 & 0.954 & 85 & 1.348 & 100 \\
\hline & 4 & 0.815 & 85 & 1.216 & 100 \\
\hline & 8 & 0.850 & 85 & 0.822 & 100 \\
\hline \multirow{4}{*}{400} & 1 & 1.377 & 90 & 2.008 & 90 \\
\hline & 2 & 1.162 & 90 & 1.720 & 100 \\
\hline & 4 & 1.130 & 100 & 1.513 & 100 \\
\hline & 8 & 0.929 & 100 & 1.193 & 100 \\
\hline
\end{tabular}

As shown, both factors have a high impact on the performance. The selected map has a great influence on the percentage of vehicles receiving warning messages and on the warning notification time, especially when the vehicle density is low. When only 50 vehicles are simulated, warning messages reach only $5.50 \%$ of vehicles in Rome and $31.60 \%$ of vehicles in New York, where the long and regular streets allow easy propagation of the wireless signal. The system requires 1 second to reach $5 \%$, and $15 \%$ of the total number vehicles, respectively.

For higher vehicle densities, the differences between the maps are reduced, the percentage of informed vehicles increases (e.g., when 400 vehicles are simulated, warning messages reach $99.35 \%$ of the vehicles in the New York scenario, and $90.30 \%$ of the vehicles in Rome), and the system needs less time to inform the same percentage of vehicles (e.g., when 200 vehicles are simulated, the system only requires 1.5 seconds to reach $60 \%$ of the vehicles in New York, and 2.3 seconds to reach the same percentage in Rome). 


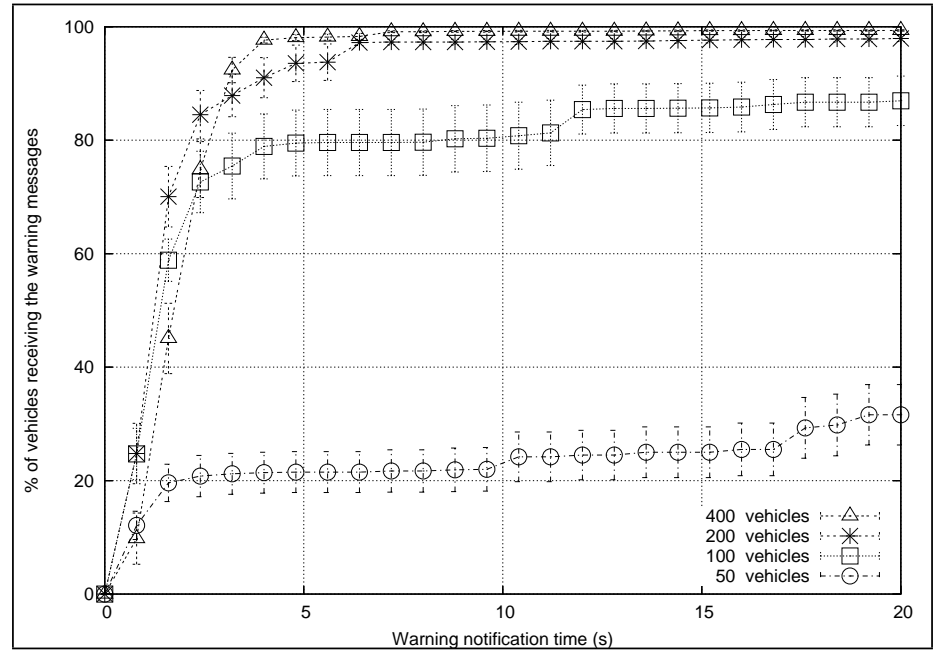

(a)

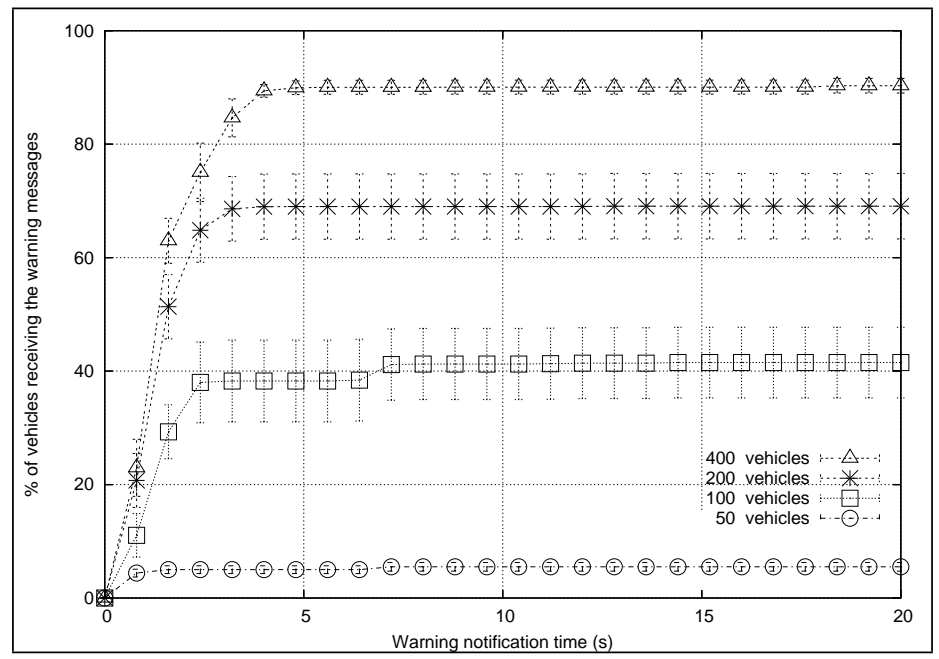

(b)

Figure 7: Warning notification time when varying the density of the vehicles and the simulated roadmap: (a) New York, and (b) Rome. 


\section{Conclusions}

In this paper we present VEACON, a Vehicle Accident Ontology for Vehicular Networks. VEACON allows to efficiently structure and encode the information collected by in-vehicle sensors, enabling the interoperatibility among all the agents involved in modern ITS (vehicles, RSUs, emergency services, authorities, etc.). VEACON combines the information sensed from the accident with the available data in the GES database to offer rich and structured information to the parties involved in traffic accidents management.

VEACON provides an ontology based approach for faster data searching and improved understanding between vehicular applications.

To verify that messages structured by using VEACON are correctly transmitted using VANETs, we performed two different tests. On the one hand, crash tests proved that the OBU correctly estimates the severity of the accident, and our system was able to collect, build, and communicate the VEACON-compliant messages with the RSU without message loss. On the other hand, by using simulations we demonstrated the feasibility of our system in terms of V2I and V2V communications. Experimental results highlight that both nearby vehicles and infrastructure elements (RSUs) are notified about and accident in just a few seconds, increasing the emergency services notification effectiveness, and thereby validating the proposed approach.

\section{Acknowledgments}

This work was partially supported by the Ministerio de Ciencia e Innovación, Spain, under Grant TIN2011-27543-C03-01.

\section{References}

Antolino Rivas, D., Barceló-Ordinas, J., Guerrero Zapata, M., Morillo-Pozo, J., 2011. Security on VANETs: Privacy, misbehaving nodes, false information and secure data aggregation. Journal of Network and Computer Applications 34 (6), 1942-1955.

Bakhouya, M., Gaber, J., Lorenz, P., 2011. An adaptive approach for information dissemination in Vehicular Ad hoc Networks. Journal of Network and Computer Applications 34 (6), 1971-1978. 
Berners-Lee, T., Hendler, J., Lassila, O., May 2001. The Semantic Web. Scientific American.

Breed, D. S., 02 1991. Vehicle Crush Zone Crash Sensor, Patent US 4995639.

Breed, D. S., Castelli, V., Johnson, W. C., DuVall, W. E., 12 1998. Vehicle Occupant Position and Velocity Sensor, Patent US 5848802.

Chong, M., Abraham, A., Paprzycki, M., 2005. Traffic Accident Analysis Using Machine Learning Paradigms. Informatica 29, 89-98.

Daeinabi, A., Pour Rahbar, A., Khademzadeh, A., 2011. VWCA: An efficient clustering algorithm in vehicular ad hoc networks. Journal of Network and Computer Applications 34 (1), 207-222.

Daemen, J., Rijmen, V., 2002. The Design of Rijndael: AES - The Advanced Encryption Standard. Springer-Verlag.

Di Lecce, V., Amato, A., May 2009. A multilevel interface for an intelligent transport system. In: IEEE Instrumentation and Measurement Technology Conference (I2MTC'09). pp. 1397-1401.

Eigner, R., Lutz, G., March 2008. Collision avoidance in VANETs - an application for ontological context models. In: Sixth Annual IEEE International Conference on Pervasive Computing and Communications (PerCom). pp. 412-416.

EuroNCAP: European New Car Assessment Programme, 2011. Evaluation procedures and test results. Available at http://www.euroncap.com/testprocedures.aspx.

Ford Motor Company, 2011. Ford researchers develop car seat that monitors drivers's heart activity. Available at http://media.ford.com/news/ fordresearchersdevelopcarseatthatmonitorsdriversheartactivity.htm.

Garshol, L. M., 2004. Metadata? Thesauri? Taxonomies? Topic maps! Making sense of it all. Journal of Information Science 30 (4), 378-391.

Gruber, T. R., December 1995. Toward principles for the design of ontologies used for knowledge sharing. International Journal Human-Computer Studies 43, 907-928. 
Husby, H. S., Simpson, L., 06 2000. Seat Belt Tension Sensor, Patent US 6081759 .

IEEE 802.11 Working Group, July 2010. IEEE Standard for Information Technology - Telecommunications and information exchange between systems - Local and metropolitan area networks -Specific requirements - Part 11: Wireless LAN Medium Access Control (MAC) and Physical Layer (PHY) specifications Amendment 6: Wireless Access in Vehicular Environments.

Kannan, S., Thangavelu, A., Kalivaradhan, R., July 2010. An intelligent Driver Assistance System (I-DAS) for vehicle safety modelling using ontology approach. International Journal Of UbiComp (IJU) 1 (3), 1-13.

Krajzewicz, D., Rossel, C., 2007. Simulation of Urban MObility (SUMO). Centre for Applied Informatics (ZAIK) and the Institute of Transport Research at the German Aerospace Centre, Available at http://sumo.sourceforge.net/index.shtml.

Martinez, F. J., Fogue, M., Coll, M., Cano, J.-C., Calafate, C. T., Manzoni, P., July 2010a. Assessing the Impact of a Realistic Radio Propagation Model on VANET Scenarios Using Real Maps. In: 9th IEEE International Symposium on Network Computing and Applications (NCA). Boston, USA, pp. 132-139.

Martinez, F. J., Toh, C.-K., Cano, J.-C., Calafate, C. T., Manzoni, P., summer 2010b. Emergency services in future intelligent transportation systems based on vehicular communication networks. IEEE Intelligent Transportation Systems Magazine 2 (2), $6-20$.

National Highway Traffic Safety Administration (NHTSA), 2011. National Automotive Sampling System. Available at http://www.nhtsa.gov/NASS.

Petzold, S., 05 1999. Rain Sensor System for a Motor Vehicle, Patent US 5900821.

RCAR, the Research Council for Automobile Repairs, 2011. Design guides and information for manufacturers. Available at http://www.rcar.org. 
Zhai, J., Wang, Q., Lv, M., July 2008a. Application of XML Topic Maps to knowledge navigation and information retrieval for urban traffic information portal. In: 27th Chinese Control Conference (CCC). pp. 458-462.

Zhai, J., Zhou, Z., Shi, Z., Shen, L., 2008b. An integrated information platform for intelligent transportation systems based on ontology. In: Xu, L., Tjoa, A., Chaudhry, S. (Eds.), Research and Practical Issues of Enterprise Information Systems II Volume 1. IFIP International Federation for Information Processing. Springer Boston, pp. 787-796. 